\title{
Diagnostic performance of wireless fractional flow reserve: a meta-analysis
}

Wen Pan, Qing-Jun Liu

Department of Cardiology, Kunshan Hospital of Traditional Chinese Medicine, Kunshan, Jiangsu, China

Submitted: 5 July 2019

Accepted: 2 June 2020

Arch Med Sci

DOI: https://doi.org/10.5114/aoms.2020.101477

Copyright $\odot 2020$ Termedia \& Banach

\section{Abstract}

Introduction: The aim of this study was to evaluate diagnostic performance of wireless fractional flow reserve (FFR) used in patients with coronary artery disease (CAD).

Material and methods: PubMed, Cochrane Library, Embase and Clinical trial.gov databases were searched by computer search and manual retrieval. The search terms included fractional flow reserve, quantitative coronary angiography, computational fluid dynamics and coronary artery disease. The meta-analysis was conducted with Stata12.0. Clinical outcomes included accuracy, sensitivity, specificity, positive likelihood ratio $(+L R)$, negative likelihood ratio (-LR), diagnostic odds ratio (DOR) and area under the receiver operating curve.

Results: Nine studies comprising 2052 vessels were included in the present meta-analysis. The sensitivity, specificity, $+\mathrm{LR},-\mathrm{LR}, \mathrm{DOC}$ and accuracy were $87 \%$ (95\% Cl: 83-94\%), 88\% (95\% Cl: 82-92\%), 7.28 (95\% Cl: 4.78-11.08), 0.14 (95\% Cl: $0.10-0.21), 50.69$ (95\% Cl: $25.22-101.88)$ and $0.94(95 \% \mathrm{Cl}$ : $0.91-0.96)$ respectively. No significant publication bias was detected.

Conclusions: This meta-analysis suggests that the clinical performance such as accuracy, sensitivity and specificity of wireless FFR is good to detect stenotic lesions with pressure-wire measured FFR as a reference.

Key words: fractional flow reserve, quantitative coronary angiography, computational fluid dynamics, coronary artery disease.

\section{Introduction}

Coronary artery disease (CAD) is the world's most common cause of death. Currently, coronary computed tomography angiography (CCTA) is the preferred imaging examination method to evaluate coronary artery disease (CAD) noninvasively, owing to its relatively high sensitivity and negative predictive value for obstructive coronary artery disease [1-3]. However, CCTA mainly assesses the anatomic severity of vessels and is limited in functional severity assessment of coronary stenosis [4]. Fractional flow reserve (FFR) is the gold standard of dynamics of coronary artery lesions $[5,6]$ and has been widely used among patients who were suspected to have CAD [7-9]. However, in the process of FFR, the ratio of pressure between the two sides of the stenosis was obtained through advancing a pressure wire toward the stenosis during coronary angiography, which is an invasive procedure and is associated with additional risks of adverse events and higher examination costs [10].

\author{
Corresponding author: \\ Qing-Jun Liu \\ Department of Cardiology \\ Kunshan Hospital of \\ Traditional Chinese Medicine \\ No.189, West ChaoYang Road \\ Kunshan, Jiangsu, China, \\ 215300 \\ E-mail: qing-jun@yandex.ru
}


A wireless non-invasive FFR method derived from computational fluid dynamics (CFD) and 3D quantitative coronary angiography (3D-QCA) was invented to assess the hemodynamic effect of coronary stenosis on the basis of their angiographic morphology [11-14]. The wireless FFR method presents high diagnostic value for coronary artery stenosis induced by myocardial ischemia without increasing radiation dosage and provides an ideal noninvasive method for evaluation of lesion dynamics. It was demonstrated that angiography-derived FFR showed good accuracy compared with invasive FFR [15]. This meta-analysis was undertaken to evaluate the diagnostic performance of wireless FFR with pressure wire FFR as a reference among patients with CAD.

\section{Material and methods}

\section{Data sources and searches}

Databases of PubMed, Cochrane Library, EMBASE, and Clinical Trials.gov were searched until October 2018 with the keywords "Fractional Flow Reserve", "Computational Fluid Dynamic", "angiography" and "Coronary Computed Tomography". References from trials and relevant reviews were manually searched for additional trials.

\section{Study selection}

The following inclusion criteria were applied: (1) Patients were suspected or confirmed with coronary artery disease; (2) Patients were assessed by wireless FFR (FFR derived by coronary angiography (FFRangio), FFR derived by coronary CT angiography $\left(F F R_{C T}\right)$, quantitative flow ratio $(Q F R)$ ) and measured invasive FFR; (3) The true positive, false positive, true negative and false negative results were reported; (4) 0.80 of invasive FFR was used as the threshold for lesion significance. Reviews, meta-analyses, observational studies, and small-sample trials $(n<50)$ were excluded. The meta-analysis follows the Standards for Reporting of Diagnostic Accuracy (STARD) [16].

\section{Data extraction and quality assessment}

Two authors extracted relevant information from the literature independently. Baseline demographic characteristics such as sample size, age, percentage of male subjects, percentage of diabetes mellitus, percentage of hypertension and study type were extracted from eligible studies. Relevant data of true positives, false positives, true negatives and false negatives were also extracted. The Quality Assessment of Diagnostic Accuracy Studies (QUADAS-2) tool was used to assess the quality of included studies. Risk of bias was evaluated based on four aspects: patient selection, index test, reference standard, flow and timing. Applicability was evaluated based on patient selection, index test and reference standard.

\section{Date analysis}

The primary outcome of sensitivity and specificity of wireless FFR in diagnosing hemodynamically significant coronary stenosis was pooled. Secondary outcomes include $+\mathrm{LR}$, -LR, DOR and area under the receiver operating curve, along with the 95\% confidence interval $(\mathrm{Cl})$ to express the diagnostic performance. Heterogeneity was detected
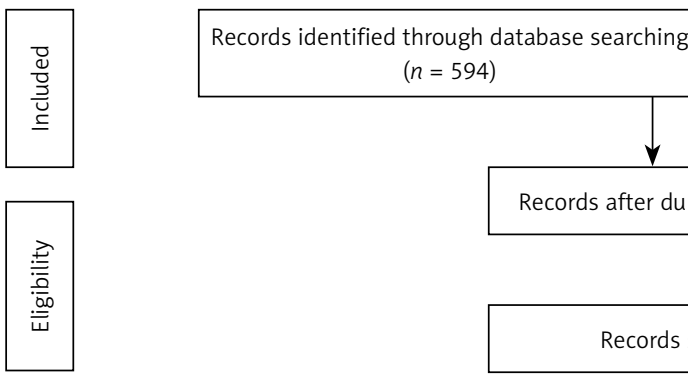

Records after duplicates removed $(n=411)$
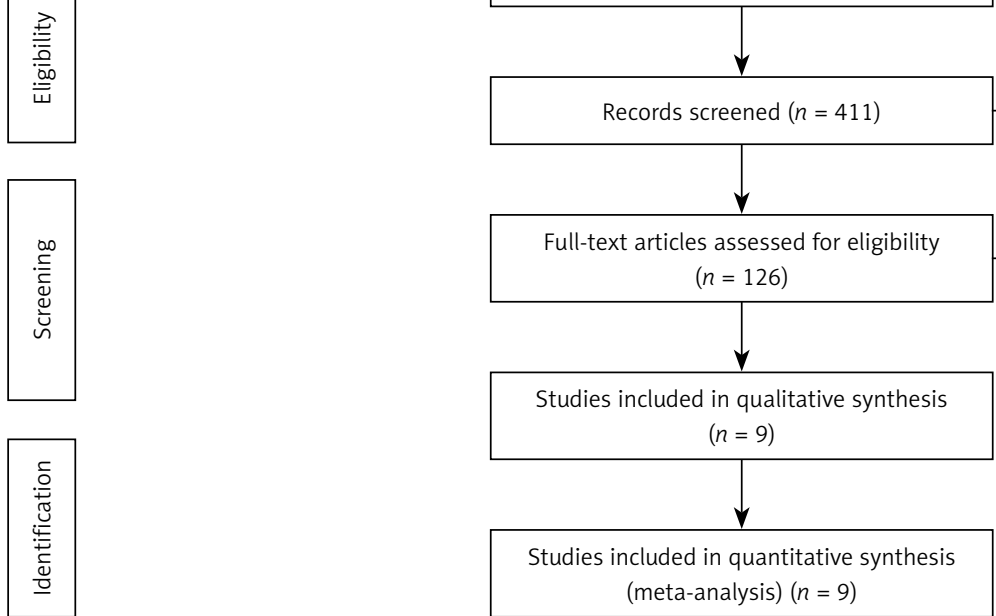

Figure 1. Flow chart showing the progress through the stages of the meta-analysis 
by bivariate boxplot and publication bias was visually inspected using a funnel plot. All analyses were performed by Stata 11.0 (StataCorp, College Station, TX, USA).

\section{Results}

\section{Literature screening and characteristics of included studies}

There were 594 articles in total found during the initial search. After removing duplicates and reviewing abstracts, finally 9 studies $[14,15,17-$ 23] comprising 2052 vessels met all inclusion criteria and were included in the analysis. The whole selection procedure was depicted in Figure 1. The baseline demographic characteristics of the included studies are detailed in Table I. Among the included 9 studies, the angiography-derived FFR calculation was performed using quantitative flow ratio (QFR) in 6 studies, $F F R_{\text {angio }}$ was used in 1 study, and FFR ${ }_{C T}$ was used in 2 studies, in which FFR was derived from the computed tomography angiography (Table I). As the included studies were diagnostic test accuracy studies, the Quality Assessment of Diagnostic Accuracy Studies (QUADAS-2) tool was used to assess the study quality. The quality assessment results are presented in Supplementary Figure S1 and Supplementary Figure S2. There was low risk of bias regarding the index test, reference standard and flow and timing. Nevertheless, there existed unclear risk of bias with respect to patient selection due to the absence of consecutive inclusion in 44\% (4/9) of the studies. In addition, low risk was observed regarding the index test, reference standard and selection of patients.

\section{Clinical results}

\section{Specificity and sensitivity}

The diagnostic result of wire FFR was used as a reference in calculation of the specificity and sensitivity of wireless FFR. The pooled sensitivity of the wireless FFR in stenotic lesion detection was 87\% (95\% Cl: 83\%-94\%) and pooled specificity was $88 \%$ (95\% Cl: 82-92\%), as shown in Figure 2.

\section{Positive and negative likelihood ratio}

Positive likelihood refers to the ratio of the true positive rate to the false positive rate, with the gold standard as a reference. Negative likelihood ratio refers to the ratio of the false negative rate to the true negative rate, with the gold standard as a reference. The diagnostic result by pressure wire FFR was the gold standard. Pooled positive likelihood (+LR) was 7.28 (95\% Cl: 4.78-11.08) and negative likelihood ratio (-LR) was 0.14 (95\% Cl: 0.10-0.21) (Figure 3).

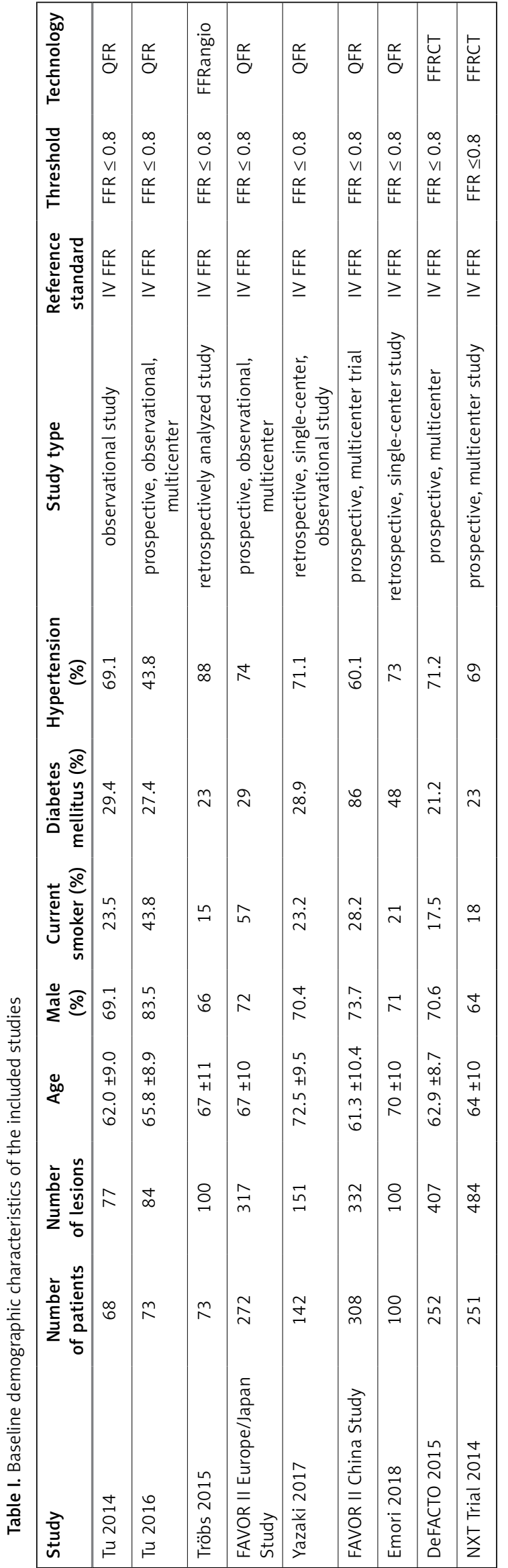




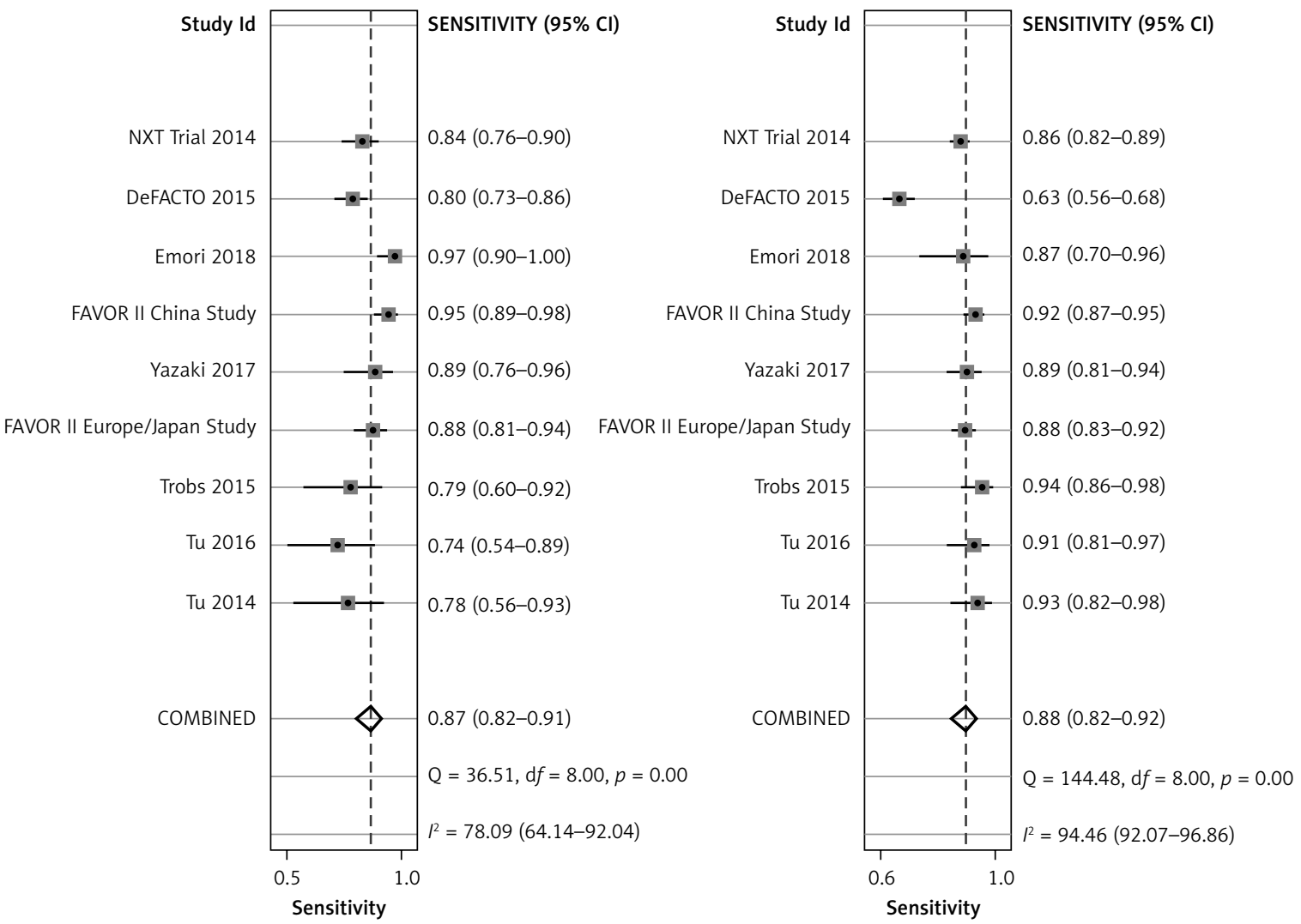

Figure 2. Individual studies and summary point estimates for sensitivity and specificity

\section{Diagnostic odds ratio and summary receiver operator curves}

Diagnostic odds ratio (DOR) refers to the ratio of $+L R$ to $-L R$, indicating the accuracy of the diagnostic test. The higher DOR is, the more accurate is the diagnostic test. The receiver operating characteristics (ROC) curve graphically presented the association between sensitivity and specificity. The closer the area under the ROC is to 1.0, the more accurate the diagnostic test will be. Summary receiver operator curve (SROC) refers to using one ROC curve to show the association between specificity and sensitivity obtained by a series of studies in a meta-analysis. The pooled DOR in our analysis was 50.69 (95\% Cl: 25.22-101.88), as shown in Figure 4. The SROC revealed an area under the curve (AUC) of $0.94(95 \% \mathrm{Cl}: 0.91-0.96)$, as shown in Figure 5.

\section{Sensitivity and publication bias analysis}

Sensitivity analysis was conducted with bivariate boxplot (Supplementary Figure S3). The sensitivity analysis result showed that the study DeFACTO 2015 is a major source of heterogeneity. According to the result of publication bias analysis performed with Deeks' funnel plot ( $p=$ $0.39)$, there was no significant publication bias, as shown in Supplementary Figure S4.

\section{Discussion}

In the last 20 years, FFR has gradually been recognized as standard functional assessment metrics, especially for critical lesions (diameter stenosis 30-70\%) [24]. The DEFER study and FAME study confirmed that percutaneous coronary intervention guided by FFR can reduce medical costs and unnecessary re-revascularization $[25,26]$. Usage of FFR for patients who have already been selected to perform $\mathrm{PCl}$ can reduce the number of stents needed to implant and improve the relative clinical effect [27-29]. However, its invasiveness and high cost limited its wide application. Seeking a non-invasive, economic, accurate alternative is necessary.

This meta-analysis showed that the diagnostic performance of wireless FFR has high sensitivity and specificity with pressure wire FFR as a reference in stable coronary artery disease patients. The pooled diagnostic accuracy of wireless FFR was high, with an AUC of 0.94 (95\% Cl: 0.66-0.94), and the high sensitivity and specificity confirm its good diagnostic performance. The high $+\mathrm{LR}(7.28$ (95\% Cl: 4.78-11.08)), low -LR (0.14 (95\% Cl: 0.10-0.21)) and high DOR (50.69 (95\% Cl: 25.22101.88)) provide strong evidence of the usefulness of wireless FFR in clinical practice. A recent published meta-analysis including 1842 vessels showed a pooled sensitivity of $89 \%$, specificity of 

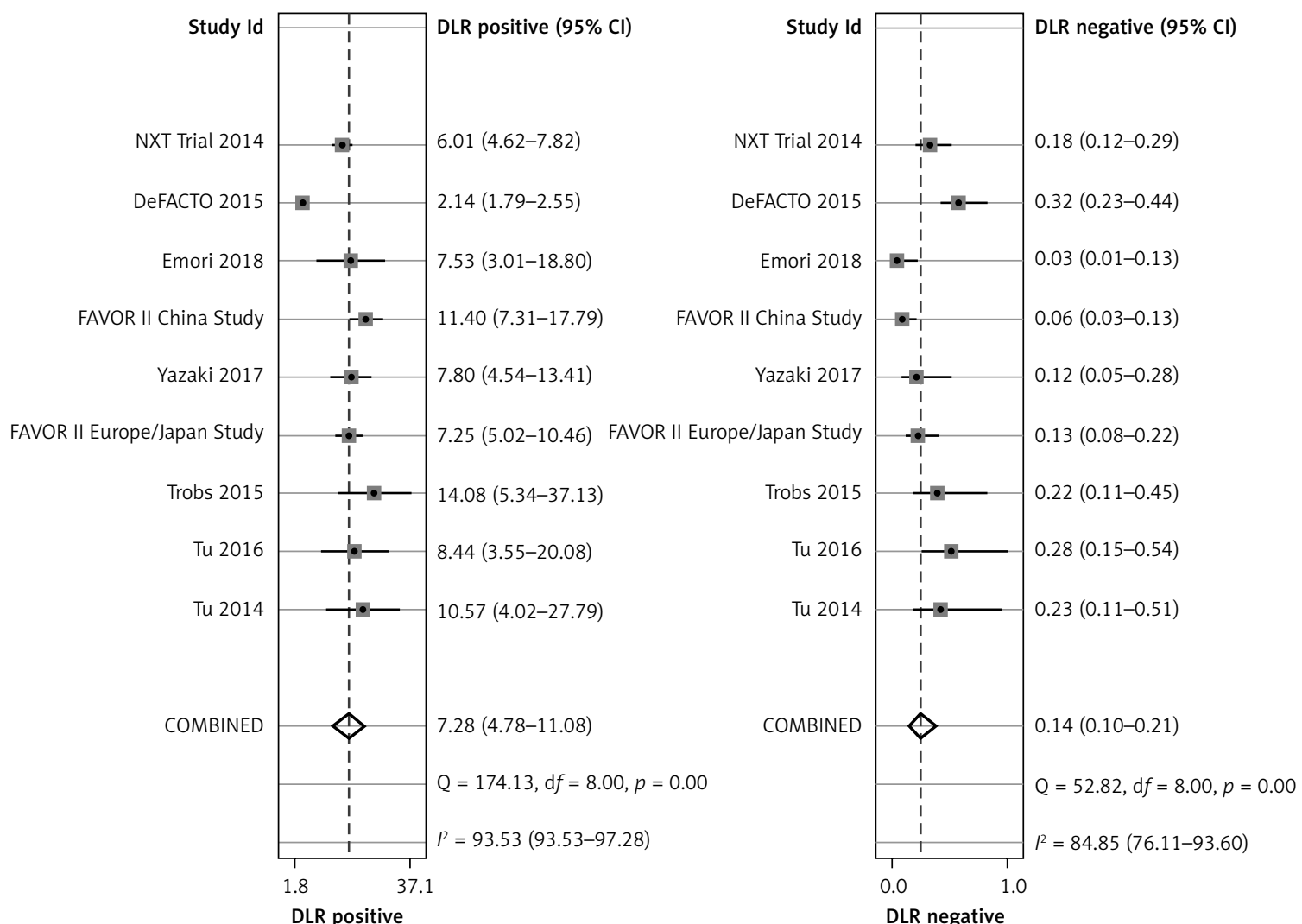

Figure 3. Individual studies and summary point estimates for $+L R$ and $-L R$
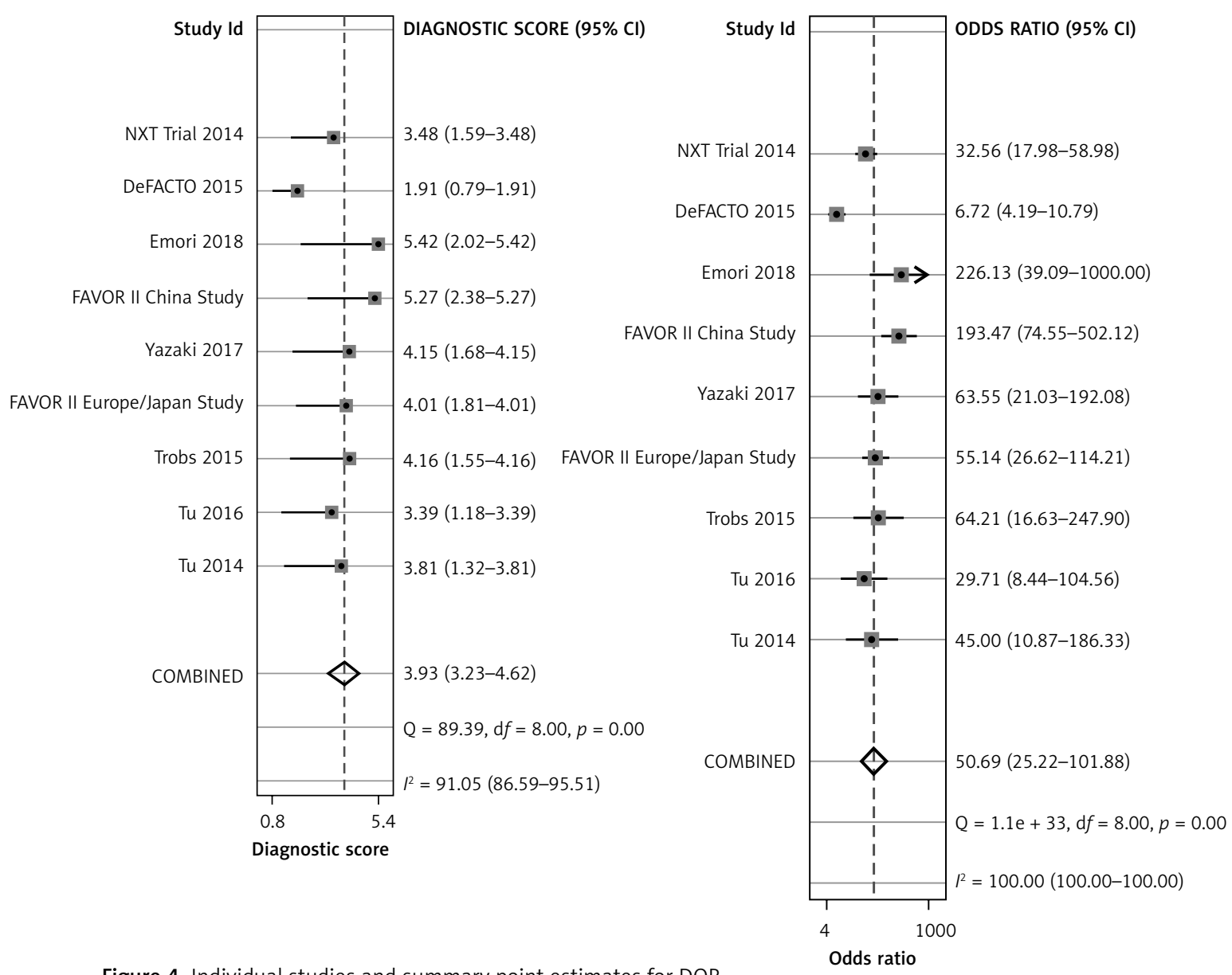

Figure 4. Individual studies and summary point estimates for DOR 


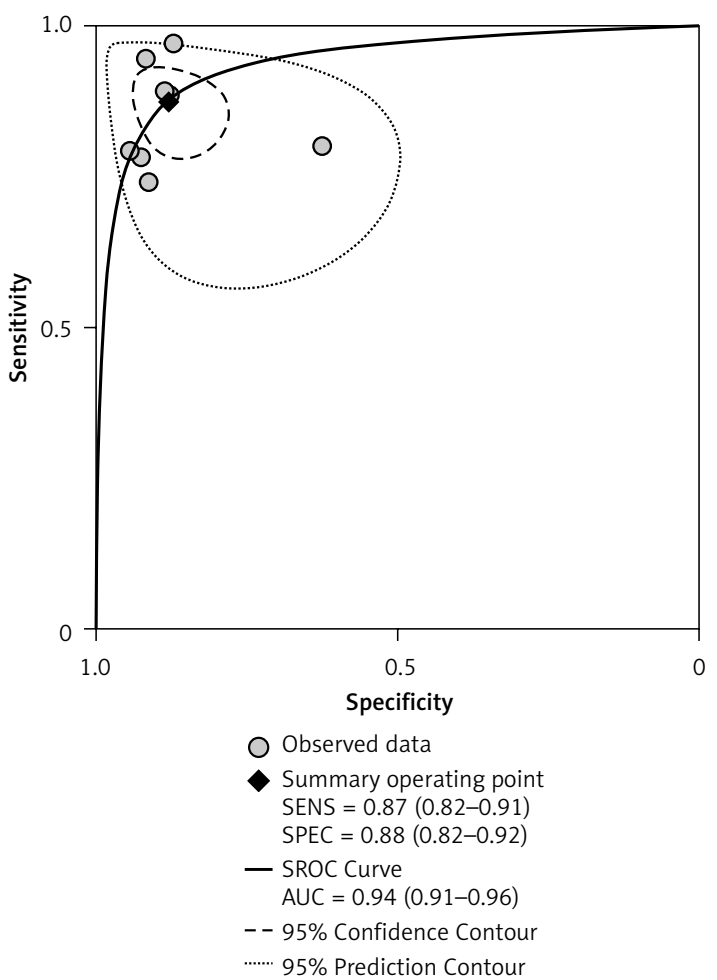

Figure 5. Summary receiver operator curves (SROC)

$90 \%$, positive likelihood ratio of 9.3 , negative likelihood ratio of 0.13 , and summary area under ROC of 0.84 [30]. Our analysis result was consistent with this meta-analysis, suggesting that wireless FFR might be good to detect hemodynamically significant lesions.

Compared with pressure wire measured FFR, wireless FFR can reduce the risk of adverse events related to the invasive pressure wire application, such as vessel dissection and myocardial infarction [31, 32]. At the same time, wireless FFR can reduce the hospitalization costs by avoiding the use of pressure wire and the related adverse events.

The advantages of our analysis include the following: this is one of very few meta-analyses evaluating the accuracy of wireless FFR with invasive FFR as a reference; most updated clinical trials were included in our analysis; $F F R_{C}$, a new type of wireless FFR in which FFR was derived from the computed tomography angiography (CTA) was included; a threshold of 0.80 by pressure wire measurement of FFR was made.

Nevertheless, there were some limitations of our meta-analysis. Firstly, most of the included studies used wireless FFR in simple lesions; thus there is a lack of application in complex lesions such as CABG and calcified lesions. Secondly, different software of the wireless FFR used in different clinical trials may lead to heterogeneity between studies. Thirdly, we could not obtain data of individual patients to address some unresolved problems to make further analysis, such as the clinical performance in the uncertainty zone. In future, more rigorous, large-sample, high quality international trials are needed; several angiography-based methods have been developed to derive FFR [12, 15, 33-34], so subgroup analysis based on various methods could be performed.

In conclusion, this meta-analysis indicates that the clinical performance such as accuracy, sensitivity and specificity of wireless FFR is good to detect stenotic lesions with pressure-wire measured FFR as a reference.

\section{Conflict of interests}

The authors declare no conflict of interest.

\section{References}

1. Miller JM, Rochitte CE, Dewey M, et al. Diagnostic performance of coronary angiography by 64 -row CT. N Engl J Med 2008; 359: 2324-36.

2. Westwood ME, Raatz HD, Misso K, et al. Systematic review of the accuracy of dual-source cardiac CT for detection of arterial stenosis in difficult to image patient groups. Radiology 2013; 267: 387-95.

3. Yang L, Zhou T, Zhang R, et al. Meta-analysis: diagnostic accuracy of coronary $\mathrm{CT}$ angiography with prospective ECG gating based on step-and-shoot, Flash and volume modes for detection of coronary artery disease. Eur Radiol 2014; 24: 2345-52.

4. Ahmadi A, Kini A, Narula J. Discordance between ischemia and stenosis, or PINSS and NIPSS: are we ready for new vocabulary. JACC Cardiovasc Imaging 2015; 8: 111-4.

5. Toth G, Hamilos M, Pyxaras S, et al. Evolving concepts of angiogram: fractional flow reserve discordances in 4000 coronary stenoses. Eur Heart J 2014; 35: 2831-8.

6. Levine GN, Bates ER, Blankenship JC, et al. 2011 ACCF/ AHA/SCAI Guideline for Percutaneous Coronary Intervention. A report of the American College of Cardiology Foundation/American Heart Association Task Force on Practice Guidelines and the Society for Cardiovascular Angiography and Interventions. J Am Coll Cardiol 2011; 58: e44-122.

7. Curzen N, Rana O, Nicholas Z, et al. Does routine pressure wire assessment influence man- agement strategy at coronary angiography for diagnosis of chest pain? The Ripcord Study. Circ Cardiovasc Interv 2014; 7: 248-55.

8. Van Belle E, Rioufol G, Pouillot C, et al. Outcome impact of coronary revascularization strategy reclassificatio with fractional flow reserve at time of diagnostic angiography: insights from a large French multicenter fractional flow reserve registry. Circulation 2014; 129 : 173-85.

9. Park SJ, Ahn JM, Park GM, et al. Trends in the outcomes of percutaneous coronary intervention with the routine incorporation of fractional flow reserve in real practice. Eur Heart J 2013; 34: 3353-61.

10. Ford ES, Roger VL, Dunlay SM, Go AS, Rosamond WD. Challenges of ascertaining national trends in the incidence of coronary heart disease in the United States. J Am Heart Assoc 2014; 3: e001097.

11. Morris PD, van de Vosse FN, Lawford PV, Hose DR, Gunn JP. "Virtual" (computed) fractional flow reserve: 
current challenges and limitations. JACC Covdiovasc Interv 2015; 8: 1009-17.

12. Morris PD, Ryan D, Morton AC, et al. Virtual fractional flow reserve from coronary angiography: modeling the significance of coronary lesions: results from the VIRTU-1 (VIRTUal Fractional Flow Reserve from Coronary Angiography) study. JACC Cardiovasc Interv 2013; 6: 149-57.

13. Papafaklis MI, Muramatsu T, Ishibashi Y, et al. Fast virtual functional assessment of intermediate coronary lesions using routine angiographic data and blood flow simulation in humans: comparison with pressure wire-fractional flow reserve. Eurolntervention 2014; 10: 574-83.

14. Tu S, Barbato E, Koszegi Z, et al. Fractional flow reserve calculation from 3-dimensional quantitative coronary angiography and TIMI frame count: a fast computer model to quantitative the functional significance of moderately obstructed coronary arteries. JACC Cardiovasc Interv 2014; 7: 768-77.

15. Tu S, Westra J, Yang J, et al. Diagnostic accuracy of fast computational approaches to derive fractional flow reserve from diagnostic coronary angiography: the international multicenter FAVOR pilot study. JACC CardiovasC Interv 2016; 9: 2024-35.

16. Bossuyt PM, Reitsma JB, Bruns DE, et al.; Standards for Reporting of Diagnostic Accuracy. The STARD statement for reporting studies of diagnostic accuracy: explanation and elaboration. Ann Intern Med 2003; 138: W1-12.

17. Nørgaard BL, Leipsic J, Gaur S, et al. Diagnostic performance of noninvasive fractional flow reserve derived from coronary computed tomography angiography in suspected coronary artery disease: the NXT trial (Analysis of Coronary Blood Flow Using CT Angiography: Next Steps). J Am Coll Cardiol 2014; 63: 1145-55.

18. Park HB, Heo R, Hartaigh BÓ, et al. Atherosclerotic plaque characteristics by $\mathrm{CT}$ angiography identify coronary lesions that cause ischemia: a direct comparison to fractional flow reserve. JACC Cardiovasc Imaging 2015; 8: 1-10.

19. Emori H, Kubo T, Kameyama T, et al. Quantitative flow ratio and instantaneous wave-free ratio for the assessment of the functional severity of intermediate coronary artery stenosis. Coron Artery Dis 2018; 29: 611-7.

20. Xu B, Tu S, Qiao S, et al. Diagnostic accuracy of angiography-based quantitative flow ratio measurements for online assessment of coronary stenosis. J Am Coll Cardiol 2017; 70: 3077-87.

21. Yazaki K, Otsuka M, Kataoka S, et al. Applicability of 3-dimensional quantitative coronary angiography-derived computed fractional flow reserve for intermediate coronary stenosis. Circ J 2017; 81: 988-92.

22. Westra J, Andersen BK, Campo G, et al. Diagnostic performance of in-procedure angiography-derived quantitative flow reserve compared to pressure-derived fractional flow reserve: the FAVOR II Europe-Japan Study. J Am Heart Assoc 2018; 7: e009603.

23. Tröbs $M$, Achenbach $S$, Röther J, et al. Comparison of fractional flow reserve based on computational fluid dynamics modeling using coronary angiographic vessel morphology versus invasively measured fractional flow reserve. Am J Cardiol 2015; 117: 29-35.

24. Consensus Committee of Clinical Application of Coronary Flow Fraction Reserve. Consensus on clinical application of coronary flow fraction reserve. Chin J Cardiol 2016; 44: 292-7.

25. Pijls NH, van Schaardenburgh P, Manoharan G, et al. Percutaneous coronary intervention of functionally nonsig- nificant stenosis: 5-year follow-up of the DEFER study. J Am Coll Cardiol 2007; 49: 2105-11.

26. Tonino PA, Fearon WF, De Bruyne B, et al. Angiographic versus functional severity of coronary artery stenoses in the FAME study fractional flow reserve versus angiography in multivessel evaluation. J Am Coll Cardiol 2010; 55: 2816-21.

27. Tonino PA, De Bruyne B, Pijls NH, et al. Fractional flow reserve versus angiography for guiding percutaneous coronary intervention. N Engl J Med 2009; 360: 213-24.

28. Pijls NH, van Schaardenburgh P, Manoharan G, et al. Percutaneous coronary intervention of functionally nonsignificant stenosis. 5-Year follow-up of the DEFER study. J Am Coll Cardiol 2007; 49: 2105-11.

29. Johnson NP, Toth GG, Lai D, et al. Prognostic value of fractional flow reserve: linking physiologic severity to clinical outcomes. J Am Coll Cardiol 2014; 64: 1641-54.

30. Collet C, Onuma Y, Sonck J, et al. Diagnostic performance of angiography-derived fractional flow reserve: a systematic review and Bayesian meta-analysis. Eur Heart J 2018; 39: 3314-21.

31. Al-Lamee R, Thompson D, Dehbi HM, et al. Percutaneous coronary intervention in stable angina (ORBITA): a double-blind, randomized controlled trial. Lancet 2018; 391: 31-40.

32. Ahmed N, Layland J, Carrick D, et al. Safety of guidewire-based measurement of fractional flow reserve and the index of microvascular resistance using intravenous adenosine in patients with acute or recent myocardial infarction. Int J Cardiol 2016; 202: 305-10.

33. Trobs M, Achenbach S, Rother J, et al. Comparison of fractional flow reserve based on computational fluid dynamics modeling using coronary angiographic vessel morphology versus invasively measured fractional flow reserve. Am J Cardiol 2016; 117: 29-35.

34. Kornowski R, Lavi I, Pellicano M, et al. Fractional flow reserve derived from routine coronary angiograms. J Am Coll Cardiol 2016; 68: 2235-7. 